\title{
Distribution of costameric proteins in normal human ventricular and atrial cardiac muscle.
}

\author{
Debora Di Mauro', Roberto Gaeta 2 , Alba Arco', Demetrio Milardi', \\ Salvatore Lentini ${ }^{2}$, Michele Runci ${ }^{1}$, Giuseppina Rizzo' ${ }^{1}$, Ludovico Magaudda ${ }^{1}$ \\ ${ }^{1}$ Department of Biomorphology and Biotechnologies, \\ ${ }^{2}$ Department of Clinical and Experimental Medicine and Pharmacology, University of Messina
}

\begin{abstract}
In the mature heart, the intercalated disc and costameres provide the cell-cell and cell-matrix junctions respectively. Intercalated disc is situated at the bipolar ends of the cardiomyocytes and the myofibrils are anchored at this structure. The costameres mediate integration with the extracellular matrix that covers individual cardiomyocytes laterally. Costameres are considered as "proteic machinery" that appears to comprise two protein complexes: the dystrophin-glycoprotein complex (DGC) and the vinculin-talin-integrin system. There are structural differences between atrial and ventricular myocytes, but there have been relatively few studies that have analyzed costameres and focal adhesion function in cardiac cells. Our previous study carried out only on atrial myocytes, demonstrated that the DGC and talin-vinculin-integrin complexes had a costameric distribution that, unlike skeletal muscle, it localized only on the I band. We performed a further immunohistochemical analysis extending also the evaluation to the normal human cardiac muscle fibers obtained from ventricle and interventricular septum, in order to define the distribution and the spatial relationship between the proteins of the two complexes also in the other heart districts. Immunoconfocal microscopy of cardiac tissue revealed the costameric distribution of DGC and of vinculin-talin-integrin system, the association of all tested proteins in intercalated disks, in disagreement with other Authors, and in T-tubule with irregular spokelike extensions penetrating toward the center of the cell. Moreover, our data showed that all tested proteins colocalize between each other.
\end{abstract}

Key words: atrium,ventricle, costameres, intercalated disk, T-tubules, immunohistochemistry,

\section{Introduction}

Cardiac myocytes are under continuous mechanical stress and the signal transduction pathways that are activated in response to mechanical forces include many unique components. Cell-cell and cell-matrix junctions in cardiac myocytes are termed intercalated discs (ICDs) and costameres, respectively. The ICDs support synchronized contraction of cardiac tissue and provide a electro-mechanical coupling [1]. The gridlike structure of costameres, which encircle the myocyte perpendicular to its long axis, participates in signaling functions and reinforces mechanically the sarcolemma, thereby helping to protect muscle cells against workload-induced membrane damage [2-3]. Costameres appear to comprise two protein complexes: the dystrophin-glycoprotein complex (DGC) and

Correpspondence: D. Milardi, Dept. of Biomorphology and

Biotechnologies, Policlinico Universitario, Torre Biologica,

Via Consolare Valeria, 1 IT-98125 - Messina, Italy;

e-mail: demil74@alice.it the vinculin-talin-integrin system [4-5]. There have been relatively few and discordant studies that have analyzed costameres in cardiac cells with the well note structural differences between atrial and ventricular myocytes. In particular, about dystrophin, some Authors showed that it is not uniquely distributed at costameres and is continuously and uniformly distributed at the cytoplasmic surface of the peripheral plasma membrane [6], while Kaprielian et al. [7] have documented that dystrophin partially colocalizes with costameric vinculin in myocytes and it is absent from ICDs. Therefore, dystrophin also localizes at the Ttubules in cardiac muscle in contrast to its known absence in skeletal muscle T-tubules [8]. Our previous study carried out only on atrial myocytes, demonstrated that the DGC and talin-vinculin-integrin complexes had a costameric distribution and both complexes are present at the level of T tubules and ICDs [9]. The aim of this study is a further immunohistochemical analysis on normal human cardiac muscle fibers obtained from ventricle and interventricular septum in order to define 

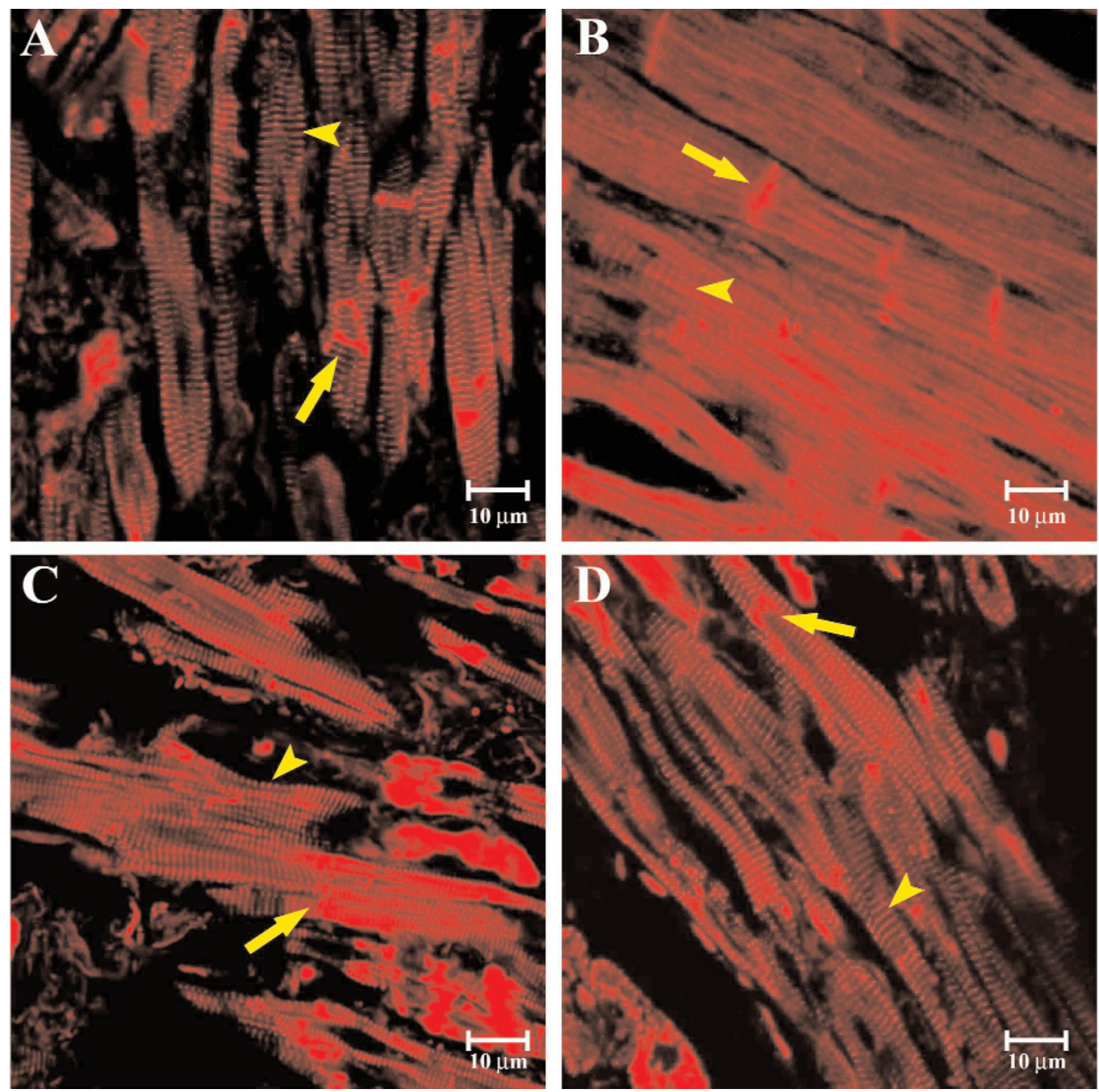

Fig. 1. Cryosections labelled with antibodies to $\alpha$-sarcoglycan (A), $\beta$-dystroglycan (B), dystrophin (C) and talin (D) showing the labelling at ICDs (arrows) and costameres (arrowed).

the localization and the relationship between the proteins of the two complexes at the level of ICDs, costameres and $\mathrm{T}$ Tubules also in the other heart districts.

\section{Materials and methods}

Normal human biopsies of left ventricle and interventricular septum were obtained from patients who underwent surgery after informed consent. They were fixed in 3\% paraformaldehyde in a $0.2 \mathrm{M}$ phosphate buffer at $\mathrm{pH} 7.4$, infiltrated with saccharose at $12 \%$ and at $18 \%$, and frozen in liquid nitrogen. Ten-mm-thick cryosections were permeabilized with $0.1 \%$ TRITON X-100 in PBS for 10 mins. Samples were pre-incubated for $1 \mathrm{hr}$. in blocking solution (PBS plus $1 \%$ bovine serum albumin-BSA and $10 \%$ goat serum-GS). In order to define a model of distribution of DGC and vinculin-talin-integrin system proteins, we analyzed all proteins with both single and double immunolabeling techniques on longitudinal and transverse sections, with different series of single and double localizations. Antibodies used were: $\alpha-, \beta-, \delta-, \gamma-$ and $\varepsilon$-sarcoglycan, diluted $1: 100,1: 200,1: 50,1: 100$, and $1: 100$ respectively (Novocastra Laboratories Ltd), $\alpha$-, and $\beta$-dystroglycan, both diluted 1:100, and dystrophin diluted 1:20 (Novocastra Laboratories Ltd), vinculin and talin both diluted 1:100 (Sigma-Aldrich). In all single immunolabellings, TRITC-conjugated IgG anti-mouse in goat (1:100; Jackson ImmunoResearch Laboratories) was used; for double-localization reactions, sections were incubated with a second antibody conjugated with FITC conjugated secondary $\operatorname{IgG}$, as second fluorochrome (1:100 dilution; Jackson ImmunoResearch Laboratories). Sections were then observed and photographed using a Zeiss LSM 5 META laser scanning microscope (Carl Zeiss).

\section{Results and discussion}

The results of present study showed that all tested proteins had costameric distribution and a constant localization in T-tubule and ICDs too. Moreover, doubleimmunofluoresce showed that all tested proteins of the 

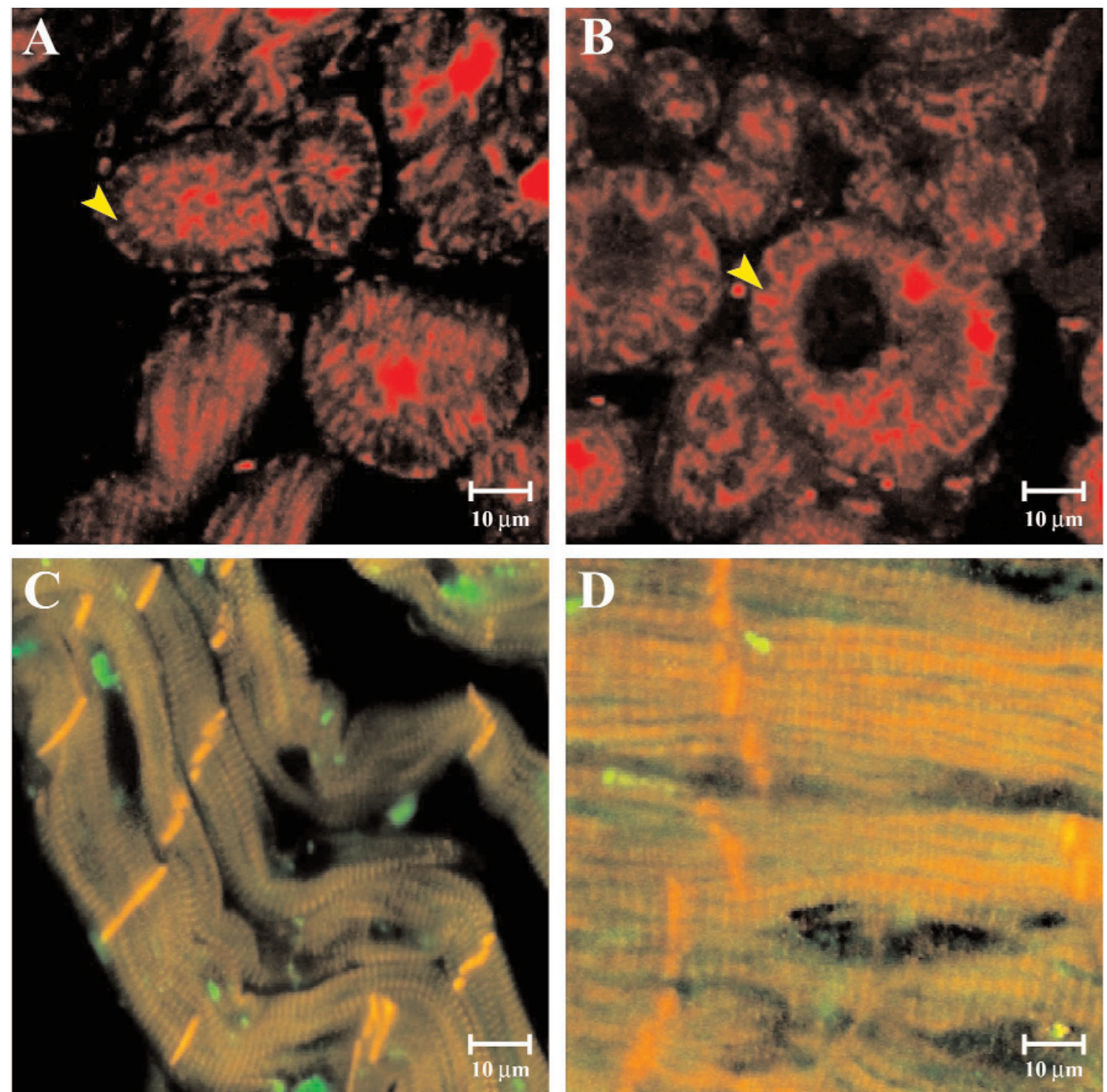

Fig. 2. Cryosections labelled with antibodies to vinculin (A) and $\alpha$-dystroglycan (B). Merged immunofluorescence images of cryosections labelled with $\varepsilon$-sarcoglycan (red)/ $\beta$-dystroglycan (green) (C) and talin (red)// $\alpha$-sarcoglycan (green) (D).

two systems, colocalized with each other. Single immunolabelling on longitudinal sections of all tested proteins (Fig. 1), evidenced their presence in ICDs (arrows) and costameres (arrowed). In transverse sections, all tested proteins showed irregular spokelike extensions of the myocyte sarcolemma, penetrating toward the center of the cell that indicated T-tubules (arrowed). In the double-localization images, a clear yellow fluorescence revealed the colocalization of all tested proteins in all different myocardial districts.

The concentration of these proteins over the costameric regions of the human cardiac fibers of ventricle and intreventricular septum, coincides with that previously observed in the atria, making to hypothesize that there are no functional differences among the present proteins in the atria and those of the ventricle or septum. It is evident the presence of tested proteins additional junctional domains in comparison to the costameres, even if remains still to appraise their role in intercalated disks or $\mathrm{T}$ tubules. It is possible to hypothesize that in the ICDs, they can provide to a structural and functional integration of the myofibrils together to the junctional systems of the same disk. Regarding T-tubules our results also showed the presence of all tested proteins implying an additional role the proteins in the E-C coupling.

\section{References}

[1] Clark KA, McElhinny AS, Beckerle MC, Gregorio CC. Striated muscle cytoarchitecture: an intricate web of form and function. Annu Rev Cell Dev Biol. 2002;18:637-706. 
[2] Pardo JV, D'Angelo Siliciano J, Craig SW. A vinculin-containing cortical lattice in skeletal muscle: transverse lattice elements ("costameres") mark sites of attachment between myofibrils and sarcolemma. Proc Natl Acad Sci USA. 1983; 80:1008-1012.

[3] Danowsky BA, Imanaka-Yoshida K, Sanger JM, Sanger JW. Costameres are sites of force transmission to the substratum in adult rat cardiomyocytes. $J$ Cell Biol. 1992;118:1411-1420.

[4] Anastasi G, Amato A, Tarone G et al. Distribution and localization of vinculin-talin-integrin system and dystrophin-glycoprotein complex in human skeletal muscle. Cells Tissues Organs. 2003;175:151-164.

[5] Samarel AM. Costameres, focal adhesions, and cardiomyocyte mechanotransduction. Am J Physiol Heart Circ Physiol. 2005;289:H2291-H2301.
[ 6] Stevenson S, Rothery S, Cullen MJ, Severs NJ. Dystrophin is not a specific component of the cardiac costamere Circ Res. 1997;80(2):269-80.

[ 7] Kaprielian RR, Severs NJ. Dystrophin and the cardiomyocyte membrane cytoskeleton in the healthy and failing heart. Heart Fail Rev. 2000;5:221-238.

[ 8] Klietsch R, Ervasti JM, Arnold W, Campbell KP, Jorgensen AO. Dystrophin-glycoprotein complex and laminin colocalize to the sarcolemma and transverse tubules of cardiac muscle. Circ Res. 1993;2:349-60.

[ 9] Anastasi G, Cutroneo G, Gaeta R et al. Dystrophin-glycoprotein complex and vinculin-talin-integrin system in human adult cardiac muscle Int. J Mol Med. 2009;23:149-159.

Submitted: 16 January, 2009 Accepted after reviews:????? 